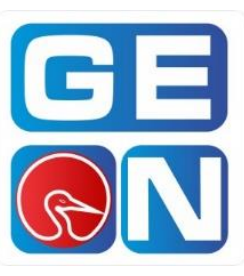

Revista GEON (Gestión, Organizaciones y Negocios.) ISSN: 2346-3910 en línea

revistageon@unillanos.edu.co

Universidad de los Llanos

Colombia

Ocañas Gallardo, Eunice Sarai ${ }^{i}$.

Un análisis de la brecha salarial por género: caso aplicado a una empresa de manufactura en

Escobedo, México

Revista GEON, Vol. 6, No. 2, 2019

Pág. 82-89

Disponible en: https://doi.org/10.22579/23463910.173

${ }^{\mathrm{i}}$ https://orcid.org/0000-0003-0817-3013

Esta publicación se encuentra bajo licencia: Creative Commons

ReconocimientoNoComercialSinObraDerivada 4.0 Internacional

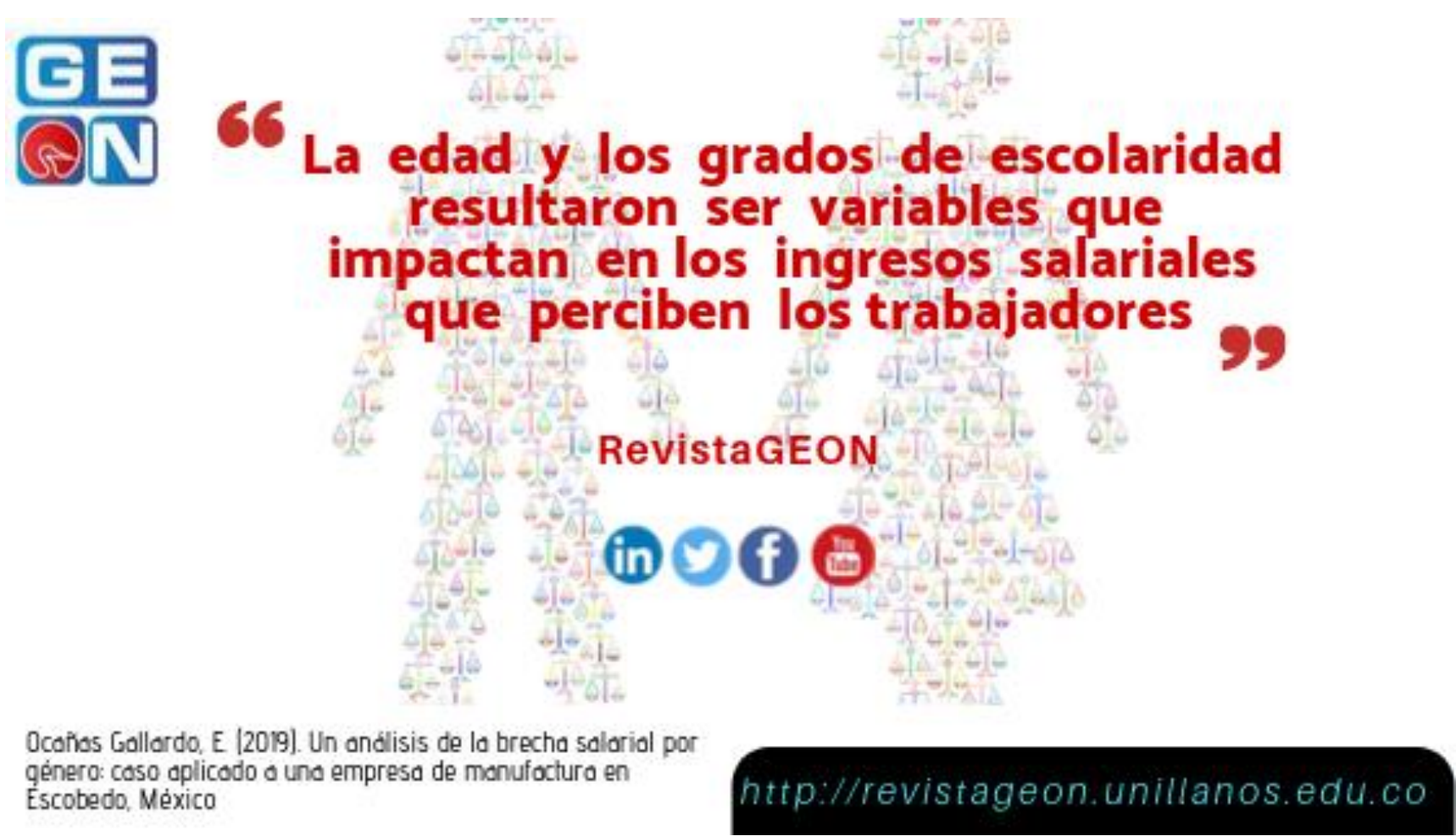




\section{Un análisis de la brecha salarial por género: caso aplicado a una empresa de manufactura en Escobedo, México}

\section{An analysis of the wage gap by gender: case applied to a manufacturing company in Escobedo, Mexico}

\section{Eunice Sarai Ocañas Gallardo ${ }^{1}$}

Cómo citar este artículo / To reference this article:
Ocañas Gallardo, E. (2019). Un análisis de la brecha salarial por género: caso aplicado a una empresa de manufactura en Escobedo, México. Revista GEON (Gestión, Organizaciones Y Negocios), 6(2), 82-89. Recuperado a partir de http://revistageon.unillanos.edu.co/index.php/geon/article/view/173
Artículo de investigación:

Fecha de recepción: 2019/05/12

Fecha de aceptación: 2019/06/30

Esta publicación se encuentra bajo licencia: Creative Commons ReconocimientoNoComercialSinObraDerivada 4.0 Internacional.

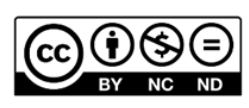

\section{Resumen}

Este artículo presenta una caracterización de los empleados de una empresa manufacturera ubicado en el municipio de Escobedo en el estado de Nuevo León, México, con datos de un levantamiento de 114 trabajadores en el primer trimestre del año 2019, se efuctua un análisis de varianza (ANOVA) de las características de los individuos. Los resultados muestran que existe una diferencia entre los ingresos que perciben las mujeres con respecto a los hombres, y esto se debe en gran medida a los grados de escolaridad y puestos que ocupan dentro de la empresa.

Palabras claves: brecha salarial, género, empresa manufacturera.

\begin{abstract}
This article presents a characterization of the employees of a manufacturing company located in the municipality of Escobedo in the state of Nuevo León, Mexico, with data of an uprising of 114 workers in the first quarter of the year 2019, an analysis of variance is effected (ANOVA) of the characteristics of the individuals. The results show that there is a difference between the income that women receive with respect to men, and
\end{abstract}

\footnotetext{
${ }^{1}$ Maestra en Administración de Empresas, Universidad Autónoma de Nuevo León, México, sarai.ocanas@gmail.com, https://orcid.org/0000-0003-0817-3013
} 
this is largely due to the levels of schooling and positions they occupy within the company.

Keywords: salary gap, gender, manufacturing company.

\section{Introducción}

En México se ha producido un fuerte incremento en el número de mujeres trabajadores, debido a un incremento de la población activa femenina, y por otra parte al incremento considerable de sus tasas de ocupación, pero aún existen diferencias en la participación en el mercado laboral entre hombres y mujeres (Godinho y Balçao, 1993).

Por su parte, Fuentes, Palma y Montero, (2005) y Arceo y Campos, (2014) señalan que la situación que ha tenido la mujer en el mercado laboral ha ido mejorando constantemente durante las últimas décadas, pero a pesar de esto aún existen diferencias con respecto a la de los hombres. Este fenómeno es muy común a nivel mundial, no obstante México necesita seguir avanzando en el tema de igualdad en el empleo.

Bajo este contexto, el municipio de Escobedo en Nuevo León en México, no es ajeno a este escenario, ya que existe un problema en el que la mujer y el hombre a pesar de su desarrollo académico y laboral no se les brinda un salario proporcional a esos conocimientos y a las aportaciones que ellos brindan en su puesto de trabajo, por lo que no garantiza en ambos géneros que se esté realizando una retribución correcta por desempeñar sus funciones dentro de las organizaciones del municipio.
En este sentido, el presente artículo analiza los diferentes factores (individuales o socioeconómicos) que pueden influir en la disminución de la brecha salarial por género. Para ello, se realiza un comparativo de estas características por género, y a través de un análisis de varianza (ANOVA) se cuantifica si existen diferencias entre hombres y mujeres.

\section{Contexto teórico}

El nivel educativo de las personas sobresale como un factor estratégico para impulsar el mejoramiento de la condición social de la mujer y del hombre, promoviendo relaciones más equitativas e igualitarias entre ambos, y contribuir a lograr una mejor calidad de vida de la población, además de brindar conocimientos para desarrollar destrezas y habilidades, la educación puede involucrar valores fundamentales que propicien el desarrollo integral de las personas, fortalezcan su dignidad, fomenten el afán de logro y superación personal y abran nuevas opciones y perspectivas de vida (Schultz, 1960; Briceño, 2011).

Bajo estos ideales, el presente artículo trata de investigar con un estudio de caso dentro de una empresa manufacturara ubicada en el municipio de General Escobedo en Nuevo León, mediante la aplicación de encuestas al personal de diversos departamentos, para 
identificar la manera en que están distribuidos sus salarios, ya que las mujeres están participando cada vez más en el trabajo remunerado y al tener mayor nivel de estudios y más experiencia laboral, esto induciría una reestructuración importante en sus remuneraciones y en sus preferencias respecto del tipo de ocupación en que se emplean o que se les ofrece. Aunque no necesariamente se va reflejado una numeración por igual con su contraparte, lo hombres.

\section{Materiales y métodos}

En la presente investigación se estableció una encuesta como instrumento de recolección de datos para entrevistar a los empleados de la empresa de manufactura que se encuentra en el municipio de General Escobedo en Nuevo León. El proceso que se siguió fue realizar un muestreo no probabilístico el cual nos dio la oportunidad para seleccionar libremente a los empleados a entrevistar, siempre y cuando cubrieran el perfil deseado, es decir, que fueran personas que tienen más de dos años laborando en la empresa y que tuvieran entre 18 a 60 años.

La encuesta se divide en dos apartados: el primer bloque captura las características individuales de los empleados en la empresa de estudio, tales como: edad, escolaridad, puesto que desempeña, estado civil, ingreso, etcétera. Mientras que en un segundo bloque se pregunta sobre la percepción que tienen acerca de su empresa y trabajo, específicamente sobre la manera en que cada uno de los empleados se ve a largo plazo de acuerdo con los salarios que tienen en la actualidad dentro de la organización y la manera en cómo perciben que podrían llegar a tener un incremento. El último apartado se realiza con base a la escala tipo Likert, con el fin de proporcionarnos una mayor facilidad y evaluar que tanto interés siente el empleado que tiene la empresa en su crecimiento laboral dentro de la organización y misma que utilizara como parámetros: siempre, algunas veces, por lo regular, casi nunca y nunca.

El instrumento aplicado en el presente estudio fue validado por expertos en el tema. Asimismo, se midio el grado fiabilidad con el Alfa de Cronbach dando como resultado 0.876 .

La población que se consideró para este estudio fueron los empleados de la empresa manufacturera, la cual cuenta con un total de 160 trabajadores en todos sus áreas o departamentos de trabajo. El nivel de confianza que se determinó utilizar fue de $95 \%$, con un error muestral de $5 \%$ y una heterogeneidad del $50 \%$, se utilizó la fórmula para poblaciones finitas (Mendenhall, Sincich y Boudreau, 1996).

Fórmula para poblaciones finitas:

$$
\mathrm{n}=\frac{\mathrm{N} * \mathrm{Z} \mathbf{a}^{2} * \mathbf{p} * \mathbf{q}}{\mathrm{d}^{2} *(\mathrm{~N}-1)+\mathrm{Z} \mathbf{a}^{2} * \mathbf{p} * \mathbf{q}}
$$

Donde " $N$ " representa la población de donde se obtendrá la muestra (160), "Z $\alpha$ " significa el nivel de confianza (1.96), " $p$ " se refiere a la proporción esperada (0.5), "q" es la proporción esperada menos 1 y por último "d" representa el margen de error (0.05), por consiguiente, el resultado fue de 114 trabajadores a encuestar. 
Es conveniente mencionar que en este estudio sólo se analiza la primera parte del cuestionario, donde se aplica un ANOVA de un factor para diversas variables de control (edad, escolaridad, estado civil y experiencia) que nos permiten cuantificar si existen diferencias significativas entre hombres y mujeres con respecto al ingreso salarial que perciben cada uno de estos.

\section{Resultados}

Los resultados del ANOVA para la muestra total (es decir, se incluye a hombres y mujeres) son presentados en el
Cuadro 1, donde se observa que las únicas variables que resultaron ser significativas en las diferencias entre grupos por género fue la edad y el ingreso salarial que perciben.

Para el caso de la edad se aprecia que existe una diferencia el auxiliar y el líder de celda, así como también de este último con el profesional o que tiene carrera. Asimismo, se aprecia que existe una diferencia entre salarios dados los puestos que se ocupan en la empresa, específicamente de la gerencia con respecto a los demás puestos.

\section{Tabla 1. Pruebas Post Hoc de ANOVA (muestra global)}

\begin{tabular}{|c|c|c|c|c|c|}
\hline Variable & pendiente & & Diferencia de & Error & Sig. \\
\hline Edad & Auxiliar & Gerencia & -6.688 & 4.032 & .464 \\
\hline & & $\begin{array}{l}\text { Líder de } \\
\text { celda }\end{array}$ & $-8.500^{*}$ & 3.048 & .048 \\
\hline & & Operario & 3.241 & 4.271 & .942 \\
\hline & & Profesional & 4.355 & 2.347 & .347 \\
\hline & Líder de & Auxiliar & $8.500^{*}$ & 3.048 & .048 \\
\hline & celda & Gerencia & 1.813 & 4.572 & .995 \\
\hline & & Operario & 11.741 & 4.784 & .109 \\
\hline & & Profesional & $12.855^{*}$ & 3.186 & .001 \\
\hline & Profesional & Auxiliar & -4.355 & 2.347 & .347 \\
\hline & & Gerencia & -11.043 & 4.137 & .065 \\
\hline & & $\begin{array}{l}\text { Líder de } \\
\text { celda }\end{array}$ & $-12.855^{*}$ & 3.186 & .001 \\
\hline & & Operario & -1.114 & 4.371 & .999 \\
\hline Ingreso & Gerencia & Auxiliar & $16562.500^{*}$ & 1623.830 & .000 \\
\hline & & $\begin{array}{l}\text { Líder de } \\
\text { celda }\end{array}$ & $15750.000^{*}$ & 1841.250 & .000 \\
\hline & & Operario & $17125.000^{*}$ & 2200.715 & .000 \\
\hline & & Profesional & $17525.000^{*}$ & 1666.354 & .000 \\
\hline & Líder de & Auxiliar & 812.500 & 1227.500 & .964 \\
\hline & celda & Gerencia & $-15750.000^{*}$ & 1841.250 & .000 \\
\hline & & Operario & 1375.000 & 1926.935 & .953 \\
\hline
\end{tabular}




\begin{tabular}{|c|c|c|c|c|}
\hline \multicolumn{2}{|l|}{ Variable dependiente } & \multirow{2}{*}{$\begin{array}{l}\begin{array}{l}\text { Diferencia de } \\
\text { medias (I-J) }\end{array} \\
1775.000\end{array}$} & \multirow{2}{*}{$\begin{array}{l}\begin{array}{l}\text { Error } \\
\text { estándar }\end{array} \\
1283.226\end{array}$} & \multirow{2}{*}{$\begin{array}{l}\text { Sig. } \\
.640\end{array}$} \\
\hline & Profesional & & & \\
\hline \multirow[t]{4}{*}{ Operario } & Auxiliar & -562.500 & 1720.378 & .997 \\
\hline & Gerencia & $-17125.000^{*}$ & 2200.715 & .000 \\
\hline & $\begin{array}{l}\text { Líder de } \\
\text { celda }\end{array}$ & -1375.000 & 1926.935 & .953 \\
\hline & Profesional & 400.000 & 1760.572 & .999 \\
\hline \multirow[t]{4}{*}{ Profesional } & Auxiliar & -962.500 & 945.141 & .846 \\
\hline & Gerencia & $-17525.000^{*}$ & 1666.354 & .000 \\
\hline & $\begin{array}{l}\text { Líder de } \\
\text { celda }\end{array}$ & -1775.000 & 1283.226 & .640 \\
\hline & Operario & -400.000 & 1760.572 & .999 \\
\hline
\end{tabular}

*. La diferencia de medias es significativa en el nivel .05.

Fuente: Elaboración propia con la corrida en el SPSS v. 24

El Cuadro 2 muestra los resultados del ANOVA para la muestra de mujeres, y se aprecia que existe una diferencia marcada entre las edades que ocupan las mujeres en los diferentes cargos de la empresa.
Además, la variable de ingreso salarial es igual en el puesto de auxiliar y profesional, es decir, una mujer con carrera profesional gana lo mismo que una auxiliar.

Tabla 2. Pruebas Post Hoc de ANOVA (muestra mujeres)

\begin{tabular}{llllll}
\hline Variable dependiente & & $\begin{array}{l}\text { Diferencia de } \\
\text { medias (I-J) }\end{array}$ & $\begin{array}{l}\text { Error } \\
\text { estándar }\end{array}$ & Sig. \\
\hline Edad & Auxiliar & Gerencia & $-13.487^{*}$ & 2.350 & .000 \\
& & Profesional & $-4.385^{*}$ & 1.246 & .003 \\
\cline { 2 - 6 } & Gerencia & Auxiliar & $13.487^{*}$ & 2.350 & .000 \\
& & Profesional & $9.103^{*}$ & 2.237 & .001 \\
\cline { 3 - 6 } & Profesional & Auxiliar & $4.385^{*}$ & 1.246 & .003 \\
& & Gerencia & $-9.103^{*}$ & 2.237 & .001 \\
\hline Ingreso & Auxiliar & Gerencia & $-17641.026^{*}$ & 1983.733 & .000 \\
& & Profesional & -423.077 & 1052.033 & .915 \\
\cline { 3 - 6 } & Gerencia & Auxiliar & $17641.026^{*}$ & 1983.733 & .000 \\
& & Profesional & $17217.949^{*}$ & 1888.458 & .000 \\
\cline { 3 - 6 } & Profesional & Auxiliar & 423.077 & 1052.033 & .915 \\
& & Gerencia & $-17217.949^{*}$ & 1888.458 & .000 \\
\hline
\end{tabular}

*. La diferencia de medias es significativa en el nivel .05.

Fuente: Elaboración propia con la corrida en el SPSS v. 24

De acuerdo con los datos recabados para los hombres, se aprecia que existe una diferencia muy marcada entre la gerencia y los demás los rangos o puestos dentro de la empresa. Sin embargo, todos los puestos con excepción de la gerencia en promedio 
ganarían de manera similar, ya que los resultados indicaron ser no significativos da pie a pensar que existen similitudes en las remuneraciones percibidad por los trabajadores.

Por último, aunque no menos importante se hace un comparativo de la variable ingreso salarial por género en la empresa de estudio. Y se encuentra que los hombres ganan o perciben mayor salario que las mujeres. A pesar de controlar la base recabada por edad y estado civil, los hombres ganan más que las mujeres.

\section{Tabla 3. Pruebas Post Hoc de ANOVA (muestra hombres)}

\begin{tabular}{|c|c|c|c|c|c|}
\hline \multicolumn{3}{|c|}{ Variable dependiente } & \multirow{2}{*}{$\begin{array}{l}\text { Diferencia de } \\
\text { medias (I-J) }\end{array}$} & \multirow{2}{*}{$\begin{array}{l}\begin{array}{l}\text { Error } \\
\text { estándar }\end{array} \\
2267.889\end{array}$} & \multirow{2}{*}{$\begin{array}{l}\text { Sig. } \\
.000\end{array}$} \\
\hline \multirow[t]{20}{*}{ Ingreso } & Auxiliar & Gerencia & & & \\
\hline & & $\begin{array}{l}\text { Líder de } \\
\text { celda }\end{array}$ & -117.857 & 1431.535 & 1.000 \\
\hline & & Operario & 1257.143 & 1964.049 & .968 \\
\hline & & Profesional & 257.143 & 1772.890 & 1.000 \\
\hline & Gerencia & Auxiliar & $16342.857^{*}$ & 2267.889 & .000 \\
\hline & & $\begin{array}{l}\text { Líder de } \\
\text { celda }\end{array}$ & $16225.000^{*}$ & 2430.387 & .000 \\
\hline & & Operario & $17600.000^{*}$ & 2777.585 & .000 \\
\hline & & Profesional & $16600.000^{*}$ & 2645.870 & .000 \\
\hline & Líder de & Auxiliar & 117.857 & 1431.535 & 1.000 \\
\hline & & Gerencia & $-16225.000^{*}$ & 2430.387 & .000 \\
\hline & & Operario & 1375.000 & 2149.640 & .968 \\
\hline & & Profesional & 375.000 & 1976.513 & 1.000 \\
\hline & Operario & Auxiliar & -1257.143 & 1964.049 & .968 \\
\hline & & Gerencia & $-17600.000^{*}$ & 2777.585 & .000 \\
\hline & & $\begin{array}{l}\text { Líder de } \\
\text { celda }\end{array}$ & -1375.000 & 2149.640 & .968 \\
\hline & & Profesional & -1000.000 & 2390.565 & .993 \\
\hline & Profesional & Auxiliar & -257.143 & 1772.890 & 1.000 \\
\hline & & Gerencia & $-16600.000^{*}$ & 2645.870 & .000 \\
\hline & & $\begin{array}{l}\text { Líder de } \\
\text { celda }\end{array}$ & -375.000 & 1976.513 & 1.000 \\
\hline & & Operario & 1000.000 & 2390.565 & .993 \\
\hline
\end{tabular}

*. La diferencia de medias es significativa en el nivel .05.

Fuente: Elaboración propia con la corrida en el SPSS v. 24

En otras palabras, un hombre y una mujer con la misma edad, estado civil y misma profesión, se encontró que los hombres ganan más que las mujeres. Por lo tanto, existe una brecha salarial por género.
Otro dato interesante que se puede apreciar en el Cuadro 4, para el caso de las mujeres es que una mujer con el grado de escolaridad de educación superior comparándola con una que término su carrera técnica y trabaja como auxiliar 
ganan relativamente lo mismo, lo cual implica que para el caso de las mujeres el tener un grado más de escolaridad en esta empresa de manufactura no impacta demasiado en su ingreso salarial. Además, de que a diferencia de los hombres no tienen o no pueden trabajar en dos puestos (operarias o líderes de celda), ya que estos trabajos son considerados especialmente para empleados varones.

No obstante, si se observa los ingresos promedio en cada uno de los puestos para los hombres, se aprecia que existen diferencias más marcadas en sus salarios con respecto a las mujeres.

C 4. Comparativo de ingreso salarial por género

\begin{tabular}{ll}
\hline Muestra global & \\
\hline Puesto & Ingreso (Promedio) \\
\hline Carrera (Licenciatura) & 12600 \\
Operario & 13000 \\
Auxiliar & 13562 \\
Líder de celda & 14375 \\
Gerencia & 30125 \\
Muestra Hombres & \\
Carrera (Licenciatura) & 14000 \\
Operario & 13000 \\
Auxiliar & 14257.14 \\
Líder de celda & 14375 \\
Gerencia & 30600 \\
Muestra Mujeres & \\
Carrera (Licenciatura) & 12115.38 \\
Operario & No aplica \\
Auxiliar & 11692.31 \\
Líder de celda & No aplica \\
Gerencia & 29333.33 \\
\hline Fuente: Elaboración propia con la corrida en el SPSS v. 24 &
\end{tabular}

\section{Conclusiones}

En este artículo se demuestra que las características individuales de los trabajadores de la empresa de manufactura "JT" en el municipio de General Escobedo en Nuevo León en México juegan un papel importante en la brecha salarial por género. En primera instancia se encuentra que la edad y los grados de escolaridad resultaron ser variables que impactan en los ingresos salariales que perciben los trabajadores. Sin embargo, variables como el estado civil resultaron ser no significativas. 
Se puede mostrar también, como los puestos de gerencia tienen un mayor ingreso salarial a comparación con el resto y predomina el hombre teniendo mayores ganacias en comparación con la mujer, no obstante, la brecha salarial es mínima.

Asimismo, se observa que la desigualdad entre hombres y mujeres se ve bien marcado en los puestos que ofrece la empresa, ya que existen puestos como operarios y líderes de celda que no son o no pueden ser ocupados por mujeres. Por lo tanto, en esas árteas existe una brecha salarial que no se puede cuantificar.

\section{Referencias}

Arceo Gómez, E. \& Campos Vázquez, M. (2014). Evolución de la brecha salarial de género en México. El Trimestre Económico. 81(323), 619-653. Recuperado a partir de http://www.scielo.org.mx/pdf/ete/v81n323/2448718X-ete-81-323-00619.pdf

Briceño Mosquera, A. (2011) La educación y su efecto en la formación de capital humano y en el desarrollo económico de los países. Apuntes del CENES. 30(51), 45-59. Recuperado a partir de

https://dialnet.unirioja.es/servlet/articulo?codigo $=3724527$

Fuentes, J., Palma A., \& Montero, R. (2005). Discriminación salarial por género en Chile: Una mirada global. Estudios de Economía, 32(2), 133-157. Recuperado a partir de http://repositorio.uchile.cl/bitstream/handle/2250 $/ 127768 /$ Jeanette_Fuentes.pdf?sequence $=1 \&$ isAl lowed=y

Godinho Delgado, M. \& Balçao, N. (1993). Mujer y trabajo. Nueva Sociedad. (124), 60-71. Recuperado a partir de http://biblioteca.ues.edu.sv/revistas/10701648N1 24-8.pdf

Mendenhall, W., Sincich, T. \& Boudreau, N. (1996). A second course in statistics: regression analysis: student's solutions manual. 5th ed. Prentice-Hall.

Schultz, T. (1960). Capital formation by education. The journal of political economy. 68(6). 571-583. Recuperado a partir de: https://www.journals.uchicago.edu/doi/pdfplus/1 $0.1086 / 258393$ 